\title{
Inverse transition in the dipolar frustrated Ising ferromagnet: The role of domain walls
}

\author{
Luciana Araújo Velasque, ${ }^{1, *}$ Daniel A. Stariolo, ${ }^{1,2, \dagger}$ and Orlando V. Billoni ${ }^{3, \dagger}$ \\ ${ }^{1}$ Departamento de Física, Universidade Federal do Rio Grande do Sul, Caixa Postal 15051, \\ 91501-970 Porto Alegre, Rio Grande do Sul, Brazil \\ ${ }^{2}$ National Institute of Science and Technology for Complex Systems, Caixa Postal 15051, 91501-970 Porto Alegre, Rio Grande do Sul, Brazil \\ ${ }^{3}$ Facultad de Matemática, Astronomía y Física, Universidad Nacional de Córdoba, \\ Instituto de Física Enrique Gaviola (IFEG-CONICET) Ciudad Universitaria, 5000 Córdoba, Argentina \\ (Received 25 September 2014; revised manuscript received 18 November 2014; published 1 December 2014)
}

\begin{abstract}
We present a theoretical study aimed at elucidating the origin of the inverse symmetry-breaking transition observed in ultrathin magnetic films with perpendicular anisotropy. We study the behavior of the dipolar frustrated Ising model in a mean field approximation as well as two other models with simple domain walls. By a numerical analysis we show that the internal degrees of freedom of the domain walls are decisive for the presence of the inverse symmetry-breaking transition. In particular, we show that in a sharp domain wall model the inverse transition is absent. At high temperatures the additional degrees of freedom of the extended domain walls increase the entropy of the system leading to a reduction of the free energy of the stripe phase. Upon lowering the temperature the domain walls become narrow, and the reduction of the number of degrees of freedom associated with them manifests in a reduction of entropy which eventually induces an inverse transition to the competing homogenous phase. We also show that, for a growing external field at constant temperature, the stripe width grows strongly when approaching the critical field line and diverges at the transition. These results indicate that the inverse transition is a continuous phase transition and that the domain wall profiles and the temperature have little effect on the critical behavior of the period of the domain as a function of the applied field.
\end{abstract}

DOI: 10.1103/PhysRevB.90.214408

PACS number(s): $75.70 . \mathrm{Ak}, 75.30 . \mathrm{Kz}, 75.70 . \mathrm{Kw}$

\section{INTRODUCTION}

Experiments on ultrathin ferromagnetic films of $\mathrm{Fe} / \mathrm{Cu}(001)$ have shown that, under a perpendicular magnetic field, the field versus temperature phase diagram displays inverse symmetrybreaking transitions $[1,2]$. Without the external field, these systems show low temperature phases with modulated order in the out-of-plane component of the magnetization. These phases are a consequence of the competition among exchange, dipolar, and uniaxial anisotropies and have been extensively studied due to the rich phenomenology [3-8]. For weak applied fields a finite global magnetization begins to develop; it is manifested in the appearance of an asymmetry between the two preferential directions of the out-of-plane local magnetization. At higher fields, stripelike phases lose stability and a bubblelike phase may be present before saturation when a uniformly magnetized phase sets in [9-11]. Both in experiments and in theoretical models a phase transition from a homogeneous to a modulated phase is observed upon decreasing the temperature at a fixed external field value. Interestingly, upon further lowering of the temperature a second transition is found, which corresponds to an inverse symmetry breaking, restoring spatial invariance at low temperatures $[1,2,11,12]$.

Inverse symmetry breaking (ISB) has been reported many times before. The most usual cases correspond to inverse melting or inverse freezing (see, e.g., Ref. [13] and references therein). The reentrance of a more symmetric phase from an already broken symmetry one at low temperatures can often be traced to a subtle interplay between energy and entropy, while

\footnotetext{
*1u.pos.fis@gmail.com

†daniel.stariolo@ufrgs.br

†billoni@famaf.unc.edu.ar
}

temperature or another thermodynamic control parameter is varied. As stated in Ref. [13], "inverse melting happens if, and only if, the so called "ordered" phase (crystal) admits more entropy than the "disordered" state; this may occur, e.g., if in the liquid phase some of the degrees of freedom of the elementary constituents are frozen, and melt in the crystalline phase." We will see that a similar phenomenon occurs in the ultrathin magnetic model studied in this work. Inverse melting is also observed in models of complex fluids with competing interactions $[14,15]$ and in the $J_{1}-J_{2}$ model in the square lattice [16,17]. In spin systems, inverse freezing has been reported mainly in theoretical descriptions of spin glasses and disordered models, in which frustration leads to complex entropic contributions [18-21]. In this work, we report on a detailed analysis of the ISB transition in a well-known model for ultrathin ferromagnetic films with perpendicular anisotropy, the dipolar frustrated Ising model (DFIM), and unveil the nature of the ISB phenomenon in this system. In previous work, the existence of ISB was established theoretically based on a scaling hypothesis for modulated systems [12] and confirmed and explored to some extent in a coarse-grained model with a Landau-Ginzburg type effective free energy [11]. This analysis confirmed the existence of ISB in two-dimensional models, but the phase diagram was restricted to relatively high temperatures due to the effective nature of the model. No attempt was made to explain the nature of the ISB. Here we show the mean field phase diagram of the DFIM in the whole temperature range where ISB transitions can be observed. We focus our analysis on the behavior of the "asymmetric-stripe" solutions. Although bubble solutions compete with stripes and may be thermodynamically stable in some region of the $H-T$ plane, it is shown in Ref. [11] that its domain of stability is probably restricted to a small region in the high temperature sector of the $H-T$ plane. 
Instead, at lower temperatures where the ISB phenomenon is observed, stripes with asymmetry in the net magnetization are the relevant solutions. We show that asymmetric stripes display reentrant behavior. In order to elucidate the nature of the reentrance, we analyze two other models which differ in the structure of the domain walls: a "sharp-wall" model, in which the transitions between positive and negative magnetizations in the stripe patterns are abrupt, and a "two-spin-wall" model, in which the transitions involve two spins. These models then are compared with the full mean field model, in which the domain walls, although much simpler than domain walls in real systems, can be spatially extended with a width that depends on the temperature and the magnetic field. Our main result is that the sharp-wall model does not support reentrant behavior, while the two-spin-wall model does. Then we conclude that the domain wall degrees of freedom are essential to drive the ISB transitions. We analyze in detail the energy, entropy, and magnetization contributions to the free energy and show that the enhanced entropic contribution of domain walls in the full model are responsible for the reentrant behavior. In addition, we show that the three models behave in the same way at sufficiently low temperatures, as expected, and analyze the behavior of the stripe widths and asymmetry as a function of magnetic fields. At low temperatures our results are compared with recent analytic work on a sharp model at $T=0$ showing a very good agreement with theoretical predictions [22].

The organization of this article is as follows. In Sec. II we introduce the models and discuss the mean field solution in an external field. In Sec. III A we analyze the solutions at very low temperatures and compare our results with exact results known for $T=0$ in a sharp-wall model. In Sec. III B we present the results for the $H-T$ phase diagrams. In Sec. III C we analyze the free energy contributions at low and high temperatures. We conclude in Sec. IV with a summary of the results and perspectives for future work.

\section{MODEL AND MEAN FIELD SOLUTIONS}

The dipolar frustrated Ising model in the two-dimensional square lattice, suitable to describe ultrathin films with perpendicular anisotropy, is defined by the following Hamiltonian:

$$
\mathcal{H}=-\frac{1}{2} \sum_{i, j} J_{i j} S_{i} S_{j}-H \sum_{i} S_{i},
$$

where $\left\{S_{i}= \pm 1, i=1, \ldots, N\right\}$ are $N$ Ising spins in a $L \times L=N$ two-dimensional square lattice,

$$
J_{i j}= \begin{cases}\delta-1 & \text { if } i \text { and } j \text { are nearest neighbors } \\ 0 & \text { if } i=j \\ -\frac{1}{r_{i, j}^{3}} & \text { otherwise }\end{cases}
$$

and $H$ is an homogeneous magnetic field perpendicular to the film. Because we are interested in describing thermodynamic phases with modulated magnetization profiles one needs to consider the local magnetizations $m_{i}=\left\langle S_{i}\right\rangle$. Then, the free energy of the model in the mean field approximation reads

$$
\begin{aligned}
F_{M F}= & -\frac{1}{2} \sum_{i, j} J_{i j} m_{i} m_{j}-H \sum_{i} m_{i}+\frac{k_{B} T}{2} \\
& \times \sum_{i}\left[\left(1+m_{i}\right) \ln \left(1+m_{i}\right)+\left(1-m_{i}\right) \ln \left(1-m_{i}\right)\right] .
\end{aligned}
$$

In this expression the first term on the right side is the internal energy, the second is the Zeeman term, and the last one is the entropy. For $H=0$ the ground state and the low temperature equilibrium states are known to correspond to stripe patterns of period $\lambda$. In this case, each period is composed of equal $\lambda^{+}=\lambda^{-}=\lambda / 2$ positive (negative) magnetization sites. At finite $H$ the direction parallel to the field will be favored and the stripes will continue to be equilibrium states but will become asymmetric with $\lambda^{+} \neq \lambda^{-}$and $\lambda^{+}+\lambda^{-}=\lambda$. The modulation length $\lambda$ is a function of the temperature and the magnetic field and then has to be considered as a variational parameter for the minimization of Eq. (3). We found it to be more efficient to minimize directly the free energy with respect to $\lambda+1$ parameters using a standard numerical minimizer than solving the equivalent set of $N$ coupled nonlinear state equations $\partial F / \partial m_{i}=0$. Also, because of the long range nature of the dipolar interaction, we found it useful to work with the Fourier transform of the energy term in Eq. (3). The Fourier transform of the couplings in the square lattice is given by [23]

$$
J_{k_{x}}=2 \delta\left(\cos k_{x}+1\right)-k_{x}^{2}+2 \pi\left|k_{x}\right|-\frac{2 \pi^{2}}{3}-2 \zeta(3),
$$

where we considered stripes perpendicular to the $x$ axis $\left(k_{y}=0\right)$ and $\zeta(x)$ is the Riemann zeta function.

In order to minimize the free energy we proceed as follows. Given a trial magnetization profile $\left\{m_{j}\right\}$, with $j=1,2, \ldots, \lambda$, we compute its Fourier transform

$$
m_{k_{x}}=\frac{1}{\sqrt{\lambda}} \sum_{j=1}^{\lambda} m_{j} e^{i k_{x} j}
$$

where the wave vectors take the values $k_{x}=2 \pi n / \lambda$, with $n=-\lambda / 2+1, \ldots, 0, \ldots, \lambda / 2$ for periodic boundary conditions. Then using Eq. (4) we compute the energy term of the free energy per unit of length $f_{M F}=F_{M F} / \lambda$ :

$$
u_{F M}=\frac{1}{2 \lambda} \sum_{k_{x}} J_{k_{x}}\left|m_{k_{x}}\right|^{2} \text {. }
$$

In this way long-range dipolar interactions are taken into account. The entropy term is computed directly using the $m_{j}$. Varying also the periodicity $\lambda$ we obtain the most general stripe profile that minimizes the free energy. Stripe profiles have been shown to be the ground states of the model at fixed magnetization [24]. In order to capture the influence of the domain wall structure we also analyzed two simplified profiles: one that included a sharp-wall and the other with an extended domain wall that we call the two-spin-wall model [25]. They are defined as follows. 


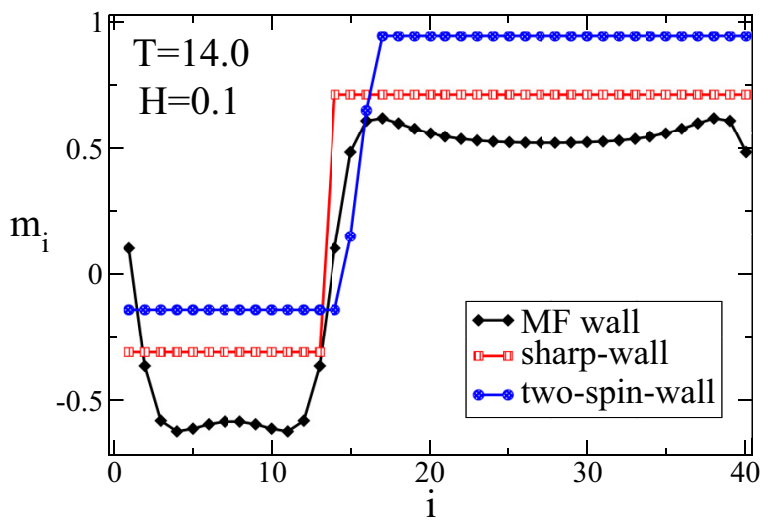

FIG. 1. (Color online) Magnetization profiles of the three models studied. The amplitudes are shifted in order to illustrate better the shape of each profile.

\section{Sharp wall}

This is a profile with an Ising-like domain wall with

$$
m_{j}= \begin{cases}m_{0} & \text { if } j \leqslant a, \\ -m_{0} & \text { otherwise }\end{cases}
$$

This profile has three parameters: the magnetization at the domains $m_{0}$, the domain wall position $a$, and the stripe pattern period $\lambda$.

\section{Two-spin wall}

In this model the domain wall consists in a couple of spins which can adjust their magnetization independently of the domain magnetization:

$$
m_{j}= \begin{cases}m_{0} & \text { if } j \leqslant a \\ m_{1} & \text { if } j=a+1 \\ -m_{1} & \text { if } j=a+2 \\ -m_{0} & \text { otherwise. }\end{cases}
$$

This model adds a new parameter with respect to the sharp-wall model, namely, the magnetization inside the domain wall $m_{1}$, i.e., this is a four parameter profile. In Fig. 1 representative profiles of the three models studied are shown.

\section{RESULTS}

\section{A. Stripe widths and asymmetry}

All our results correspond to $\delta=6$. With this value of $\delta$ we can capture the physics of the problem and make the numerical analysis feasible. At low temperatures domain walls are sharp and it is expected that all three models behave in the same way. Of particular interest is the dependence of the stripe width, or modulation length, for a fixed low temperature as a function of the magnetic field. Because the stripes become asymmetric under the influence of an external field, the dependence of the asymmetry parameter is also of interest. These functions are shown in Fig. 2 which shows the solutions of the mean field model at $T=2$. There is a critical field value, which for $T=2$ is $H_{c} \sim 0.345$, at which the stripe width diverges. One can also see that the positive component of the magnetization profile follows the growth of the stripe width

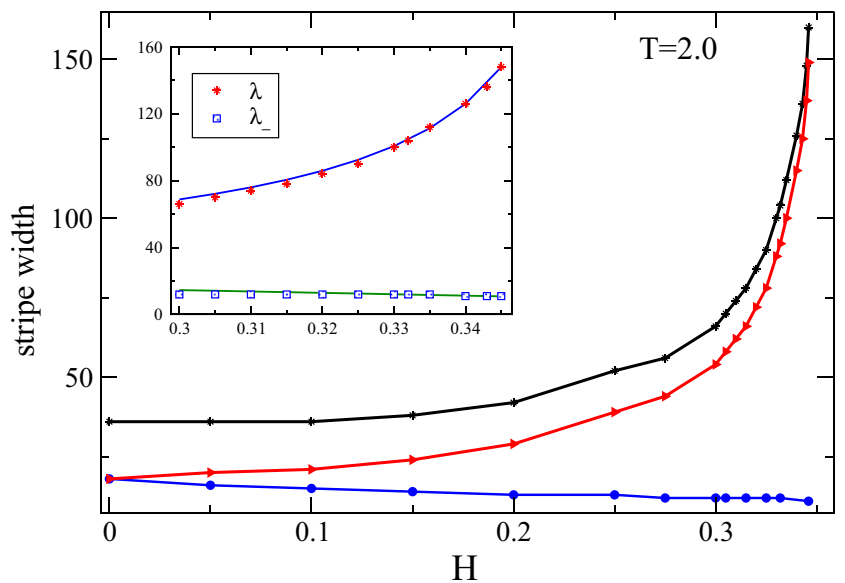

FIG. 2. (Color online) Stripe width $\lambda$ (black asterisk), positive component of magnetization $\lambda^{+}$(red triangles), and asymmetry $\lambda^{-}$ (blue circles) as functions of the magnetic field for $T=2$. Inset: Fits according to analytic predictions from Ref. [22].

and also diverges, while the asymmetry (negative component) decreases very slightly from its value at $H=0$. For $H>H_{c}$ a homogeneously magnetized solution has the minimum free energy. Nevertheless, a true divergence of the stripe width is not accessible for computational limitations on the size of the variational problem that is being solved, and then it is natural to ask what is the precise behavior of the stripe width near the critical field value. Recently, Johansen et al. [22] obtained exact results for these parameters in a model with sharp domain walls at zero temperature. In particular, they obtained that the stripe width at $T=0$ diverges as a power law:

$$
\lambda \propto\left(H-H_{c}\right)^{-1 / 2} .
$$

We expect that results from the model studied here at finite but very low temperatures should behave in the same way. In fact, a fit of our data for $T=2$ with a power law near the critical field yields very good agreement with the exact predictions for the sharp-wall model of Ref. [22] at $T=0$. The fits are shown in the inset of Fig. 2. An important conclusion is that the divergence of the stripe width at a critical value of the field implies a continuous transition from the modulated to the homogeneous phase, at variance with usual expectations. In the numerical solutions it is possible to observe that stripes with finite widths continue to exist above the critical line, and in this sense they represent metastable solutions with free energy larger than the homogenous one, with a crossing at some field value. Nevertheless, this does not imply a first-order transition since the solution with minimum free energy below the critical line corresponds to one with continuously increasing stripe width, which in fact diverges at a critical field value tending itself in a continuous way to the homogeneous solution. This trend was also observed for any temperature along the critical line shown in Fig. 3, implying a continuous transition from the modulated to the homogeneous state at the mean field level. Further evidence is provided by the continuous behavior of the magnetization at the critical field, as is shown in the analysis of Sec. III C. 


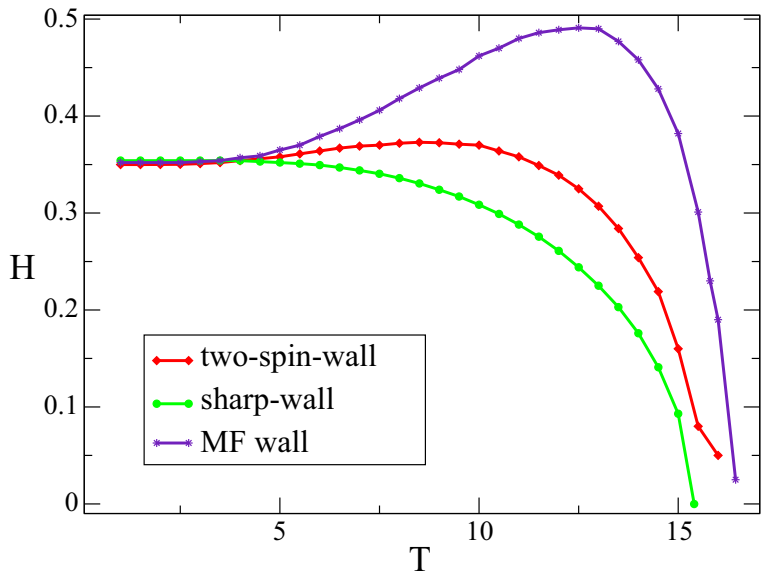

FIG. 3. (Color online) Field-temperature phase diagrams for the three models defined in the text.

\section{B. Phase diagrams}

In order to understand the nature of the interesting reentrant behavior observed in ultrathin magnetic films with perpendicular anisotropy, we have solved the complete mean field phase diagram of the DFIM for the asymmetric-stripe solutions in the field versus temperature plane for the three models defined above. The results are shown in Fig. 3. Below the curves for each model the equilibrium solution is an asymmetric stripe with a variable stripe width as discussed in the previous section. The asymmetric-stripe solutions compete with the homogeneous solution, which is locally stable for any $H$ at low $T$. At some critical field, $H_{c}$, the homogeneous solution becomes the thermodynamic one and dominates the high field section of the phase diagram. As anticipated, the low temperature behavior of the three models is the same. Nevertheless, at some point as $T$ grows, the critical field becomes different for each model, signaling different behaviors. The upper curve corresponds to the solution of the general mean field model, in which each local magnetization is considered as a variational parameter in the minimization of the free energy. The reentrant behavior is evident. In stark contrast, the sharp-wall model does not show signs of reentrant behavior; the critical field curve bends monotonically towards lower field values as $T$ grows, as seen in the Fig. 3 . What is the origin of the different behaviors of both models? As discussed previously, the profiles in both cases are very similar at low temperatures. As $T$ grows the difference must reside in the structure of the domain walls, which is the only place where additional degrees of freedom enter the scene and affect the free energy. In fact, the sharp-wall model has the simplest possible domain wall structure, a single discontinuity between two oppositely saturated regions. This is the limiting case in which the domain period becomes much larger than the wall width. At the mean field level, it is easy to see that this wall will have negligible influence on the entropy of the system. At variance with this, in the full model the walls tend to develop a nontrivial structure as temperature grows. The finite width of the walls, even at the mean field level, are enough to induce a decisive entropic contribution to the free energy of the modulated solutions. In order to further confirm if this is indeed the case, we analyze a model which is minimally different from the sharp-wall one, namely, a model in which the walls are composed of two sites with equal and opposite magnetization, i.e., one more degree of freedom with respect to the sharp wall. The result is evident in Fig. 3; this slight change in the structure of the wall is enough to induce a small reentrance in the phase diagram. Our conclusion is that the structure of the domain walls is essential to the ISB phenomenon seen in ultrathin magnetic films with perpendicular anisotropy. In the last section we analyze in detail the contributions of the energy, entropy, and external field terms to the free energy of the models.

\section{Free energy analysis}

In Figs. 4 and 5 we show the field dependence of the free energy, energy, entropy, and magnetization for the stripe solutions of the mean field and sharp-wall models together with the homogeneous solution at two characteristic temperatures,

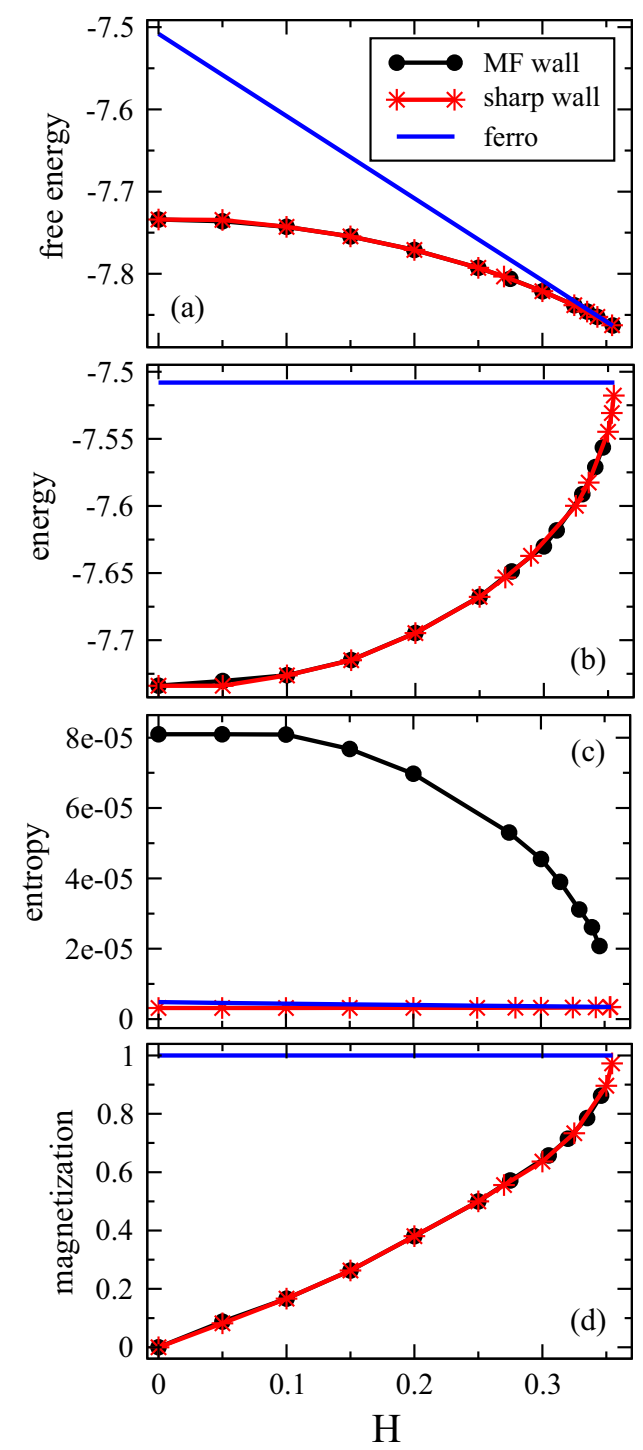

FIG. 4. (Color online) Free energy, energy, entropy, and magnetization as functions of the applied field at $T=2$. 


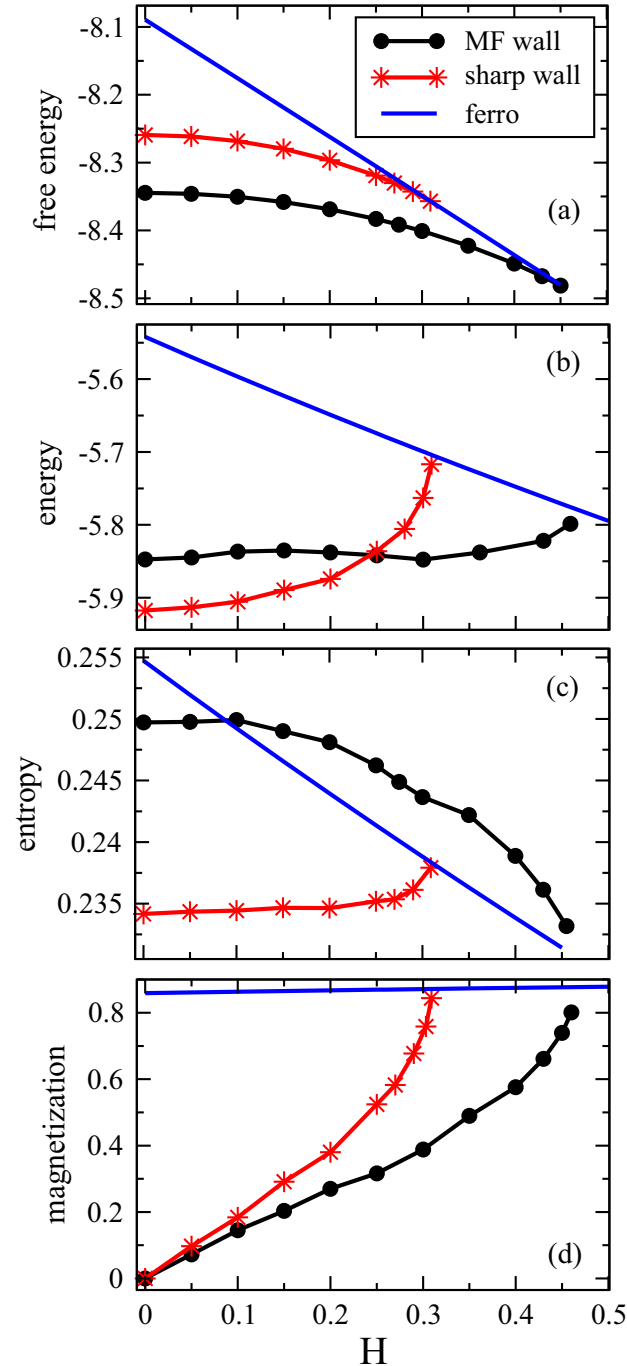

FIG. 5. (Color online) Free energy, energy, entropy, and magnetization as functions of the applied field at $T=10$.

$T=2$ and $T=10$ (see Fig. 3). A comparison of the free energy curves at both temperatures confirms what was anticipated, i.e., that the full model and the sharp-wall model behave in the same way at $T=2$, but the complete model has a lower free energy for any field value at $T=10$, i.e., in the region under the dome where the reentrant behavior is observed.

\section{1. $T=2$}

At $T=2$ thermal effects are negligible. This is reflected in particular in the almost zero value of the entropy. The magnetization confirms the expectation from the exact results of the sharp-wall model of Ref. [22]; the transition between the modulated and homogeneous states is continuous, signaled by the divergence of the stripe width at a critical value of the field. Accordingly, the magnetization grows continuously from $m=0$ at $H=0$ to $m=1$ at $H=H_{c}$.

\section{2. $T=10$}

At $T=10$ thermal effects are evident, implying a departure of both models from the common behavior seen at $T=2$. The main difference is observed in the entropy plots of Fig. 5. The mean field profiles have a notably higher entropy than those of the sharp-wall model. This is probably due to the extended nature of domain walls in the mean field model, which activates degrees of freedom not present in the sharpwall model. Particularly for small fields, the entropic advantage of the mean field model ensures a much lower free energy. At fields near the limit of stability of the sharp-wall model, this has a higher magnetization than the mean field one, but this is not enough to change the balance in the free energy, still dominated by energy and entropy contributions. As can be seen in the free energy and magnetization curves, the transition to the homogeneous state is continuous, as for $T=2$. At $H=$ $H_{c} \approx 0.46$ the stripe width diverges similarly to what happens at low temperatures. The two-spin-wall model has a behavior in between the complete and sharp-wall ones, reinforcing our interpretation of the physical picture described above.

Summarizing, the lower free energy of the extended domain wall model as compared to the sharp domain wall model is due to the combined effect of both the energy and the entropy. At low fields before the crossing of the energies of the extended domain walls and the sharp domain wall [see Fig. 5(b)], the excess of entropy manifests in a reduction of the free energy and after the crossing the reduction is due to the energy contribution. As a result of these contributions the transition line moves to higher fields.

In a work by Vindigni et al. [25] the authors showed that the mean field approximation of the DFIM is useful for the description of the temperature behavior of the stripe width of ultrathin $\mathrm{Fe}$ films epitaxially grown on $\mathrm{Cu}$, and also that this model is adequate for the description of the domain wall profiles. Our results extend the application of the model to give a microscopic explanation of the reentrance behavior observed in these systems [2].

\section{CONCLUSIONS}

Reentrance is an interesting property related to the stability of magnetic phases. We have shown that the ISB transition in ultrathin ferromagnetic films with perpendicular anisotropy occurs as a result of the additional degrees of freedom in the structure of the domain walls. We compared the phase diagrams of three models: a mean field model with extended walls, a sharp-wall model, and an intermediate two-spin-wall model. When compared with a sharp domain wall scenario the extended domain has a higher entropy, reflecting the importance of the internal degrees of freedom in the reduction of the free energy. Furthermore, our results show that with sharp domain walls the inverse transition is absent. On the other hand, the structure of the domain walls does not affect the dependence of the period of the stripes as a function of the applied field. We realized that for temperatures lower than the bump in the phase diagram, the stripe period is well described according to a zero temperature model which assumes sharp domain walls. This implies that in this range of temperatures the transition between the ferromagnetic and the stripe phase is continuous in the mean field approximation. Furthermore, our results for the mean field model indicate that the whole critical line is a line of continuous transitions. The sensitivity of the inverse transition to the internal degrees of freedom of 
the domain walls was tested through the addition of 1 degree of freedom to the sharp-wall model. The results show that this is enough to induce an inverse transition, completely absent in the sharp-wall model. The mechanism of ISB can be summarized as follow. In the low temperature range the domain wall profiles are sharp and the critical field for reaching the ferromagnetic phase is nearly constant. As the temperature increases the domain walls acquire some finite width and structure and then the entropy increases inducing a lowering of the free energy and hence a higher field is needed to enter into the homogeneous ferromagnetic phase.
It would be interesting to test these conclusions in models with realistic domain walls and quantify experimentally the extension of the reentrant phenomenon in thin films and its influence on the stability of magnetic domains.

\section{ACKNOWLEDGMENT}

This work was partially supported by CAPES/SPU through Grant No. PPCP007/2011, CONICET, SeCyT-Universidad Nacional de Córdoba (Argentina), and CNPq (Brazil).
[1] N. Saratz, A. Lichtenberger, O. Portmann, U. Ramsperger, A. Vindigni, and D. Pescia, Phys. Rev. Lett. 104, 077203 (2010).

[2] N. Saratz, U. Ramsperger, A. Vindigni, and D. Pescia, Phys. Rev. B 82, 184416 (2010).

[3] O. Portmann, A. Vaterlaus, and D. Pescia, Nature (London) 422, 701 (2003).

[4] J. Choi, J. Wu, C. Won, Y. Z. Wu, A. Scholl, A. Doran, T. Owens, and Z. Q. Qiu, Phys. Rev. Lett. 98, 207205 (2007).

[5] S. Pighn, O. V. Billoni, D. A. Stariolo, and S. A. Cannas, J. Magn. Magn. Mater. 322, 3889 (2010).

[6] A. Abanov, V. Kalatsky, V. L. Pokrovsky, and W. M. Saslow, Phys. Rev. B 51, 1023 (1995).

[7] K. De'Bell, A. B. MacIsaac, and J. P. Whitehead, Rev. Mod. Phys. 72, 225 (2000).

[8] L. Nicolao and D. A. Stariolo, Phys. Rev. B. 76, 054453 (2007).

[9] T. Garel and S. Doniach, Phys. Rev. B 26, 325 (1982).

[10] R. Díaz-Méndez and R. Mulet, Phys. Rev. B 81, 184420 (2010).

[11] S. A. Cannas, M. Carubelli, O. V. Billoni, and D. A. Stariolo, Phys. Rev. B 84, 014404 (2011).

[12] O. Portmann, A. Gölzer, N. Saratz, O. V. Billoni, D. Pescia, and A. Vindigni, Phys. Rev. B 82, 184409 (2010).
[13] N. Schupper and N. M. Shnerb, Phys. Rev. E 72, 046107 (2005).

[14] J. Pȩkalski, A. Ciach, and N. G. Almarza, J. Chem. Phys. 138, 144903 (2013).

[15] N. G. Almarza, J. Pȩkalski, and A. Ciach, J. Chem. Phys. 140, 164708 (2014).

[16] J. Yin and D. P. Landau, Phys. Rev. E 80, 051117 (2009).

[17] S. L. A. de Queiroz, Phys. Rev. E 84, 031132 (2011).

[18] A. Crisanti and L. Leuzzi, Phys. Rev. Lett. 95, 087201 (2005).

[19] M. Sellitto, Phys. Rev. B 73, 180202(R) (2006).

[20] M. Paoluzzi, L. Leuzzi, and A. Crisanti, Phys. Rev. Lett. 104, 120602 (2010).

[21] C. K. Thomas and H. G. Katzgraber, Phys. Rev. E 84, 040101 (2011).

[22] T. H. Johansen, A. V. Pan, and Y. M. Galperin, Phys. Rev. B 87, 060402(R) (2013).

[23] S. A. Pighín and S. A. Cannas, Phys. Rev. B 75, 224433 (2007).

[24] A. Giuliani, J. L. Lebowitz, and E. H. Lieb, Phys. Rev. B 80, 134420 (2009).

[25] A. Vindigni, N. Saratz, O. Portmann, D. Pescia, and P. Politi, Phys. Rev. B 77, 092414 (2008). 